\title{
BMJ Open Mortality of ethnic minority groups in the UK: a systematic review protocol
}

\author{
Fiona Stanaway (D) , ${ }^{1}$ Naomi Noguchi, ${ }^{1}$ Erin Mathieu, ${ }^{1}$ \\ Saman Khalatbari-Soltani (D) , ${ }^{1}$ Raj Bhopal ${ }^{2}$
}

To cite: Stanaway F, Noguchi N, Mathieu $\mathrm{E}$, et al. Mortality of ethnic minority groups in the UK: a systematic review protocol. BMJ Open 2020;10:e034903. doi:10.1136/ bmjopen-2019-034903

\section{- Prepublication history and} additional material for this paper are available online. To view these files, please visit the journal online (http://dx.doi. org/10.1136/bmjopen-2019034903).

Received 10 0ctober 2019 Revised 13 February 2020 Accepted 19 May 2020
Check for updates

\section{(c) Author(s) (or their} employer(s)) 2020. Re-use permitted under CC BY-NC. No commercial re-use. See rights and permissions. Published by BMJ.

${ }^{1}$ School of Public Health, University of Sydney, Sydney, New South Wales, Australia ${ }^{2}$ Usher Intsitute, University of Edinburgh, Edinburgh, UK

Correspondence to Dr Fiona Stanaway;

fiona.stanaway@sydney.edu.au

\section{ABSTRACT}

Introduction Growing ethnic diversity in the UK has made it increasingly important to determine the presence of ethnic health inequalities. There has been no systematic review that has drawn together research on ethnic differences in mortality in the UK.

Methods All types of observational studies that compare all-cause mortality between major ethnic groups and the white majority population in the UK will be included. We will search Medline (OvidSP), Embase (OvidSP), Scopus and Web of Science and search the grey literature through conference proceedings and online thesis registries. Searches will be carried out from inception to 2 August 2019 with no language or other restrictions. Database searches will be repeated prior to publication to identify new articles published since the initial search. We will conduct forward and backward citation tracking of identified references and consult with experts in the field to identify further publications and ongoing or unpublished studies. Two reviewers will independently screen studies and extract data. Two reviewers will independently assess the quality of included studies using the Newcastle-Ottawa Scale. If at least two studies are located for each ethnic group and studies are sufficiently homogeneous, we will conduct a meta-analysis. If insufficient studies are located or if there is high heterogeneity we will produce a narrative summary of results.

Ethics and dissemination As no primary data will be collected, formal ethical approval is not required. The findings of this review will be disseminated through publication in peer reviewed journals and conference presentations.

PROSPERO registration number CRD42019146143.

\section{INTRODUCTION}

Growing ethnic diversity in the $\mathrm{UK}^{1}$ and the need to comply with legislation ensuring health for all $^{23}$ has made it increasingly important to determine the presence of health differences by ethnicity. Mortality is an important measure of overall health status, and mortality differences by ethnicity and migration status are a frequent topic of research both in the UKand other countries. ${ }^{4-6}$ Despite this, there remains little consensus on how mortality differs by ethnicity in the UK, particularly in UK-born ethnic minority individuals. ${ }^{4}$ Part of the difficulty in understanding the mortality differences between
Strengths and limitations of this study

We will include all studies comparing all-cause mortality between pre-specified ethnic groups and the white majority population in the UK.

- We will conduct an extensive and sensitive search of online databases and a thorough search for unpublished studies

- We will examine the extent to which country of birth and socio-economic status contribute to ethnic inequalities in mortality.

- Lack of data on both country of birth and ethnicity in a large number of studies may reduce our ability to conduct the planned subgroup analyses comparing the health of UK-born and overseas-born ethnic minority group members.

ethnic minority groups in the UK arises from variability in how ethnicity is defined in studies, with some studies using self-reported ethnicity and others using proxy measures such as country of birth or ancestry. The use of country of birth has some limitations in that it will: (a) include people born overseas that are not a member of the ethnic group of interest such as white British born in India and (b) limit ethnic minority individuals to the overseas-born, a population in which the healthy migrant effect is an important driver of observed health differences.

The lack of consensus around mortality differences by ethnicity in the UK is also influenced by the complexity of the comparisons, as observed mortality differences will be impacted by a number of factors, including the ethnic group under study, the ethnic group being compared with and the time period. Methodological and quality differences between studies could also contribute to different findings, particularly when comparing data from cohort studies to that of the unlinked census and registry-based studies. There has also been very little exploration and as a result, limited consensus on what the underlying drivers of mortality differences by ethnicity in the UK might be. Place of birth (in particular being born 
overseas compared with being born in the UK) is likely an important predictor of mortality differences as well as socioeconomic status (SES). However, results can be conflicting for the influence of SES on the health of ethnic minority persons, particularly in terms of the relationship between SES and mortality in migrants. ${ }^{46}$

This complexity and lack of consensus underlies the importance of conducting a systematic review that draws together all of the different pieces of research on mortality differences by ethnicity in the UK and synthesises them in a rigorous manner. A systematic review will help to provide clarity on health inequalities in terms of mortality in the UK and provide guidance for policies promoting health equity.

To the best of our knowledge, there has been no other systematic review of the relationship between ethnicity and all-cause mortality in the UK. To address this gap, we have developed a protocol for a systematic review that will identify, appraise and synthesise the evidence comparing all-cause mortality rates between major ethnic groups and the white majority population in the UK.

\section{Research question}

The aim of this systematic review is to answer the following question: how do all-cause mortality rates differ between ethnic minority groups and the white majority population in the UK? The population $(\mathrm{P})$ is defined as the population of the UK, the 'risk factor' of interest (I term) is being a member of an ethnic minority population, the comparator $(\mathrm{C}$ term $)$ is being a member of the white majority population and the outcome $(\mathrm{O})$ is mortality.

\section{METHODS AND ANALYSIS}

\section{Protocol design and registration}

This protocol has been prepared according to the Preferred Reporting Items for Systematic Reviews and
Meta-Analysis Protocols statement and checklist (see online supplementary appendix 1). ${ }^{8}$ The protocol has also been registered in the PROSPERO international prospective register of systematic reviews (http://www. crd.york.ac.uk/PROSPERO) (CRD42019146143). Any amendments to the protocol will be submitted to PROSPERO to establish a record of any changes and will be reported in the final published systematic review.

\section{Patient and public involvement}

No members of the public have been involved in the research design process. We will produce plain language summaries of our results for dissemination to members of the public.

\section{Eligibility criteria \\ Population}

The population will be restricted to that of the UK and can include studies on samples in any country or region within the UK. Studies will be limited to those on populationbased samples. Studies restricted to populations with a specific disease such as diabetes will be excluded as mortality rates in these population subgroups would be higher and not able to be meaningfully combined with those based on the whole population.

\section{Ethnicity}

Ethnicity can be defined by self-report or by proxy measures such as country of birth, country of birth of parents or ancestry. Table 1 shows the ethnic groups considered for inclusion in the study. These are based on ethnicity classifications used in the 2011 censuses of England and Wales, Scotland and Northern Ireland. ${ }^{9-11}$ Ethnic groups selected for inclusion represent at least $0.5 \%$ of the population in any one of these three censuses. The exceptions are broad non-specific categories such as 'other white' and

\begin{tabular}{ll}
\hline Table 1 Ethnic groups considered for inclusion in the systematic review \\
\hline Census category ethnic group & Composite/specific ethnic groups and synonyms to be accepted \\
\hline British/white British & White \\
\hline English/white English & White \\
\hline Scottish/white Scottish & White \\
\hline Irish/white Irish & White \\
\hline Polish & Eastern European \\
\hline Indian/British Indian/Indian Scottish & South Asian \\
\hline Pakistani/British Pakistani/Pakistani Scottish & South Asian \\
\hline Bangladeshi/British Bangladeshi & South Asian/other South Asian \\
Chinese/British Chinese/Chinese Scottish & East Asian \\
African/British African/African Scottish & Black/African origin/any ethnic group with an origin from any specific sub- \\
& Saharan African country (eg, Ghanaian) \\
\hline Caribbean & Black/African Caribbean/West Indian/any ethnic group with predominantly African \\
\hline Black Irish/black Scottish/black British & ancestry from any specific Caribbean country (eg, Jamaican) \\
\hline White and black Caribbean & Black/African origin \\
\hline White and Asian & Mixed background \\
\hline
\end{tabular}


'other Western European' that were considered to be too heterogeneous to be meaningful and can have varying definitions depending on the sample. Where possible, we selected ethnic group classifications from the three UK censuses that were the most specific and narrowly defined rather than a larger composite group (eg, Indian and Pakistani British/Scottish as separate categories rather than the combined South Asian British/Scottish category). However, we selected broader groupings when more narrowly defined groupings were not available (eg, Caribbean rather than Jamaican, African rather than Ghanaian). When broader groupings are used to define ethnic categories, we have provided in the second column of table 1 details of what other more specific groupings will be included under the umbrella of this broader category. In contrast, when smaller more specific groupings are used, we provide in column two, details of what broader, less specific groupings would also be accepted in studies where only these broader groupings are used.

Studies that group multiple and extremely diverse ethnic groups together as one single category (such as all non-white ethnic minorities) will be excluded. Due to frequently observed differences in mortality between South Asians and East Asians, ${ }^{47}$ we will also not include data from studies using the composite group of Asian, where this group combines South Asians and East Asians as one category.

\section{Comparators}

The comparator group is the ethnic majority population which could include any of the following groups in the UK depending on the location of the study:

1. White British - the majority population for the UK.

2. White English/Welsh-the majority population for studies in England and Wales.

3. White Scottish—the majority population for studies in Scotland.

4. White Irish-the majority population for studies in Northern Ireland,

5. White-with all white or all white British ethnic groups included together.

6. Rest of the population-all other ethnic groups apart from the ethnic group(s) of interest in the study.

\section{Outcome}

The outcome will be the all-cause mortality rate comparison by ethnicity which can be presented as a standardised mortality ratio (SMR), relative risk (RR) or HR. Studies providing age-adjusted beta coefficients will be included and the beta coefficients exponentiated. Studies that provide absolute measures of effect will also be included if sufficient information is provided to estimate relative measures. We will include outcomes adjusted for or stratified by (a) age and sex; (b) age, sex and SES; and (c) all other confounders.

\section{Study types}

Due to the frequent under-representation of ethnic minority populations in cohort studies, ${ }^{12}$ we will include all observational study types that meet our PICO inclusion criteria. This will include:

1. Cross-sectional registry-based studies (unlinked numerator and denominator).

2. Longitudinal registry-based studies (unlinked numerator and denominator).

3. Cohort studies, including those involving data linkage.

\section{Search strategy}

We will conduct searches of Medline (OvidSP), Embase (OvidSP), Scopus and Web of Science (which includes conference proceedings). We will conduct further searches of the grey literature through EThOS (the British Library e-theses online service) and ProQuest dissertations and theses: UK and Ireland. We will additionally search NICE website and conduct searches on Google given that some material is likely to be published as government reports more readily available from the internet than in published journals. Searches will be carried out from inception to 2 August 2019 with no language or other restrictions. Database searches will be repeated prior to publication to identify new articles published since the initial search. We will attempt to contact the authors of relevant studies where additional data may be available on mortality by ethnic subgroups. We will also perform forward and backward citation tracking of identified relevant articles. We will contact experts in the field for additional studies not located as part of the comprehensive search. We will also contact chief investigators of cohort studies in the UK where data on ethnicity and mortality are likely to have been collected but have not been published. The search strategy was developed by FS in consultation with a medical librarian with expertise in conducting searches for systematic reviews. The Medline search strategy is provided in online supplementary appendix 2.

\section{Data management}

Search results will be exported into EndNote V.X8.2 for screening purposes. An Excel spreadsheet will be used to document the selection process and will document the total number of references located by each database, the total number of references identified after removal of duplicates, the total number of references identified via grey literature searches and the number of references selected at each stage of the screening process and reasons for exclusion.

\section{Selection process}

Two authors (FS and NN) will independently screen titles and abstracts for possible selection into the study. Any article identified by at least one author will be included in the list of full-text articles to review in the second stage of article selection. Two authors (FS and $\mathrm{NN}$ ) will then independently review full-text versions of articles selected in the screening stage to confirm their eligibility for inclusion. Disagreements will be resolved by consensus or when necessary, consultation with a third reviewer (RB). For studies that use overlapping datasets 
such as registry-based studies that have overlapping time periods, the study with data over the longest period will be included. If the time periods are of equivalent length, we will select the study that includes the most recent time period.

\section{Data extraction}

Data will be extracted by two authors (FS and NN) independently. Data will be entered into a data extraction form that will be pilot tested by the two authors prior to commencing data extraction. The extracted information will include: study citation, study design, study location and setting, ethnic group(s) included and method of ascertainment of ethnicity, comparison group, participant characteristics ( $n$, mean age, sex, SES) in each group, participation rates/losses/linkage rates in each group, method of outcome ascertainment, number of events in each group, the measure of effect for mortality comparison (SMR, HR, RR) and the CI or SE. We will extract the following effect measures if available: (a) SMR/HR/RR adjusted for age and stratified by or adjusted for sex; (b) HR/RR adjusted for age and SES and stratified by or adjusted for sex; (c) HR/RR adjusted for other confounders. After completion of independent data extraction, the two authors will review both sets of extracted data together to check for errors and disagreements. Any disagreements will be resolved by consensus with the help of an additional author (EM) if necessary. Finalised data will be collated into an Excel spreadsheet. For studies with missing data or with some outcome data not disaggregated by sex or particular ethnic groups, contact will be made with original authors requesting the raw data if available. Contact will also be made with investigators of major cohort studies in the UK where data on ethnicity and mortality have likely been collected but not reported in publications.

\section{Risk of bias assessment of individual studies}

Study quality will be appraised independently by two authors (FS and EM). Disagreements will be resolved by consensus or when necessary, consultation with a third reviewer (RB). The quality of included studies will be appraised using a modified version of the NewcastleOttawa Scale ${ }^{13}$ (online supplementary appendix 3). The risk of bias table will be grouped according to study type. For cross-sectional studies, only the first three questions under selection, the single question under comparability and the first question under outcome will be used. Some further specific details on the use of the Newcastle-Ottawa Scale in this systematic review are as follows:

The Newcastle-Ottawa Scale will be scored using a similar approach to the Cochrane risk of bias tool, where judgement will be made about the risk of bias being high, low or unclear. A low risk of bias will be equivalent to receiving stars for particular items as recommended in the manual for the Newcastle-Ottawa Scale. However, this modified approach will allow us to distinguish between studies that likely have a high risk of bias due to serious methodological flaws and those with unclear risk of bias due to inadequate reporting or lack of information about the likelihood of particular biases such as salmon bias ${ }^{14}$ in a particular ethnic group.

Other differences in the use of this scale will be as follows: in terms of comparability, we will only assess if the study adjusts for/stratifies by age and sex. In terms of ascertainment of exposure, the ideal method of exposure assessment will be self-reported ethnicity which will be given a low risk of bias assessment. Proxy measures for ethnicity such as country of birth will also receive a low risk of bias rating if there is good evidence that this is an accurate measure of ethnicity in the specific instance. As the accuracy of the country of birth as a proxy measure varies by the ethnic group ${ }^{15}$ in studies that include a number of different ethnic groups, judgement of risk of bias will be made for each ethnic group included in the study and provided as a supplementary file, but only summary judgement for the study overall will be displayed in the main risk of bias table. The summary judgement will be based on the average risk of bias for exposure ascertainment across all included ethnic groups.

We will add an additional domain of other bias to incorporate the problem of numerator/denominator mismatch that can occur in unlinked registry-based studies. The likelihood of this bias being present will be judged according to publications reporting the likelihood of this bias in UK data for specific ethnic groups in addition to the information provided in the included studies. For studies using data linkage, reported linkage rates will be used as part of the judgement of numeratordenominator bias. Similar to exposure ascertainment, numerator-denominator bias may differ between ethnic groups, and when this is likely, a risk of bias judgement will be made for each ethnic group separately, but only a summary judgement for the study overall will be displayed in the main table.

\section{Quality of evidence for individual ethnic groups}

We will examine the quality of the body of evidence for mortality differences for each ethnic group using GRADE criteria. ${ }^{16}$ This will include consideration of risk of bias assessment (with consideration given to the specific risk of bias assessment for individual ethnic groups in terms of exposure ascertainment, linkage rates, etc), inconsistency of results, indirectness of evidence, imprecision and publication bias.

\section{Data synthesis}

Data synthesis will be carried out by author SKS using a random effects model. ${ }^{17}$ Analyses will be conducted in STATA V.16.0 (StataCorp, College Station, Texas, USA). We will only conduct quantitative synthesis if we locate at least two studies for a particular ethnic group and if there is sufficient homogeneity to enable meaningful synthesis as detailed below in the section on subgroup analysis and investigation of heterogeneity. In the absence of a quantitative synthesis, we will provide a narrative synthesis of 
results by the ethnic group. Quantitative synthesis of ageadjusted results will be stratified by sex and ethnic group. We will conduct additional quantitative synthesis of results adjusted for age and SES also stratified by sex and ethnic group if available data permits. If data extracted are adjusted for rather than stratified by sex, we will summarise this additional data narratively and provide the results in the appendices. If extracted effect measures are adjusted for other potential confounders in addition to age, sex and SES, such as health behaviours and comorbidities, we will summarise this information narratively and provide the data in the appendices.

Given the likely diversity in measures of effects used between unlinked registry studies and cohort studies, we will treat standardised mortality ratios, HRs and relative risks as equivalent measures of effect. As event rates are likely to be low in population-based samples including in the non-exposed group, the HR and relative risk should be equivalent. ${ }^{18}$ In addition, as the proportion of most ethnic minority populations included in the analysis will range between $0.5 \%$ and $2.5 \%$, the standardised mortality ratio is less likely to be a biased representation of the relative risk as the exposure rate (ethnicity) is low. ${ }^{19}$ However, given that the standardised mortality ratio can be biased when both age-specific mortality ratios and population age distribution differ between ethnic groups, ${ }^{20}$ we will include consideration of difference in effect measures in our assessment of heterogeneity as discussed below. We will screen for publication bias using a funnel plot and Begg's test ${ }^{21}$ if at least 10 studies are located for a particular quantitative synthesis. We will also investigate publication bias by sub-group analysis comparing results of published and unpublished data if sufficient data are available.

Where data sources for a single ethnic group include disparate time periods, we will arrange studies by date and then conduct a random-effects cumulative meta-analysis ${ }^{22}$ to examine how comparative mortality estimates evolve over time. If data are available on both country of birth and self-reported ethnicity from included studies, we will conduct subgroup analyses within ethnic groups by country of birth (UK-born vs overseas-born). The need for this subgroup analysis is based on the need to account for the healthy migrant effect as a potential underlying cause of observed differences in mortality rates and the importance or understanding if there are differences in mortality between overseas-born and UK-born ethnic minority group members. We also plan subgroup analyses based on different comparison populations (eg, white British, white Scottish, white Irish). This is due to frequently poorer observed health in white Scottish and white Irish populations compared with white British populations. ${ }^{4}$ In addition, there is some evidence that the health of non-white minority groups can differ between countries in the UK. If sufficient high-quality cohort studies are located, we will also conduct sensitivity analyses restricted to these high-quality studies with results from cross-sectional studies and unlinked registry studies removed. If high heterogeneity is observed, we will investigate whether this is reduced by conducting the prespecified subgroup and sensitivity analyses listed below.

\section{Investigation of heterogeneity}

We will assess for the presence of heterogeneity using Cochran's $Q$ and the I ${ }^{2}$ Statistic. ${ }^{23}$ If we observe an $I^{2}$ value of $50 \%$ or more, we will explore possible explanations for the observed heterogeneity in subgroup and sensitivity analyses as detailed below. If sufficient studies are available, we will consider the use of meta-regression in our exploration of causes of heterogeneity. The first two subgroup analyses listed below will be conducted regardless of the presence or absence of statistical heterogeneity.

\section{Subgroup analyses}

1. UK-born versus overseas-born within each ethnic group to examine the contribution of early life environment to observed differences in mortality by ethnicity.

2. Published versus unpublished results within each ethnic group to examine the presence of publication bias.

\section{Subgroup analyses to explore heterogeneity}

1. Method of ethnicity ascertainment between studiescountry of birth versus self-reported ethnicity versus other methods.

2. Definition/included groups in one major ethnic group-for example, South Asian versus subgroups of Indian, Pakistani, and Bangladeshi.

3. Comparison population/geographic location-for example, white majority population in England and Wales versus white Scottish population in Scotland.

\section{Sensitivity analyses}

1. Study design-non-cohort studies removed.

2. Risk of bias-within cohort studies only, studies with high risk of bias will be removed.

3. Measure of effect-HR versus relative risk or standardised mortality ratio.

If any of the above are identified as a plausible explanation of the observed heterogeneity, we will conduct a quantitative synthesis at the subgroup level if sufficient studies are available. If insufficient studies are available, we will summarise the results of studies narratively.

\section{ETHICS AND DISSEMINATION}

As no primary data will be collected, formal ethical approval is not required.

To our knowledge, the proposed systematic review will be the first to systematically collect and synthesise evidence on mortality differences between the major ethnic groups in the UK. The results of the review will provide important evidence about health inequalities and provide important guidance for policies promoting health equity. It is also likely that the review will identify important gaps in the knowledge base such as a lack of research in particular ethnic groups or insufficient 
evidence in terms of differences in mortality between UK-born and overseas-born members of particular ethnic minority groups.

On completion of the review, we will implement a robust knowledge translation strategy that will include publication in peer-reviewed journals with selection of an open access format where possible, presentation of results at relevant conferences, and production of plain language summaries for dissemination of results to members of the public.

\section{Twitter Saman Khalatbari-Soltani @Saamaankh}

Contributors RB and FS conceived the idea for the study. FS, NN, SK-S, EM and RB planned and designed the study protocol in terms of ethnic groups to be included, literature search strategy, risk of bias assessment and data synthesis. FS wrote the first draft with all authors contributing critical insights and comments of specific elements (NN search strategy and data management, EM risk of bias assessment, SK-S statistical analysis and data synthesis and RB categorisation of ethnicity and need for comparison between UK-born and foreign-born populations). All authors have approved and contributed to the final version of the protocol.

Funding Open access fees were covered by financial support from the Sydney School of Public Health Academic Support Scheme.

Competing interests None declared.

Patient consent for publication Not required.

Provenance and peer review Not commissioned; externally peer reviewed.

Open access This is an open access article distributed in accordance with the Creative Commons Attribution Non Commercial (CC BY-NC 4.0) license, which permits others to distribute, remix, adapt, build upon this work non-commercially, and license their derivative works on different terms, provided the original work is properly cited, appropriate credit is given, any changes made indicated, and the use is non-commercial. See: http://creativecommons.org/licenses/by-nc/4.0/.

ORCID iDs

Fiona Stanaway http://orcid.org/0000-0003-2104-3010

Saman Khalatbari-Soltani http://orcid.org/0000-0001-8437-1906

\section{REFERENCES}

1 Office for National Statistics. Ethnicity and national identity in England and Wales: 2011, 2012. Available: https://www.ons.gov.uk/ peoplepopulationandcommunity/culturalidentity/ethnicity/articles/ ethnicityandnationalidentityinenglandandwales/2012-12-11

2 Siva N. Race relations in the UK's National health service. Lancet 2009;373:1935-6.

3 Sealy-Jefferson S, Vickers J, Elam A, et al. Racial and ethnic health disparities and the Affordable care act: a status update. J Racial Ethn Health Disparities 2015;2:583-8.
4 Bhopal RS, Gruer L, Cezard G, et al. Mortality, ethnicity, and country of birth on a national scale, 2001-2013: a retrospective cohort (Scottish health and ethnicity linkage study). PLoS Med 2018;15:e1002515.

5 Marmot MG, Adelstein AM, Bulusu L. Lessons from the study of immigrant mortality. Lancet 1984;1:1455-7.

6 Stanaway FF, Blyth FM, Naganathan V, et al. Mortality paradox of older Italian-born men in Australia: the Concord health and ageing in men project. J Immigr Minor Health 2020;22:102-9.

7 Scott AP, Timæus IM. Mortality differentials $1991-2005$ by selfreported ethnicity: findings from the ONS longitudinal study. $J$ Epidemiol Community Health 2013;67:743-50.

8 Shamseer L, Moher D, Clarke M, et al. Preferred reporting items for systematic review and meta-analysis protocols (PRISMA-P) 2015: elaboration and explanation. BMJ 2015;350:g7647.

9 Office for National Statistics. Census: ethnic group (write-in responses), local authorities in England and Wales, 2011. Available: https://www.ons.gov.uk/census/2011census [Accessed 06 Jun 2019].

10 National Records of Scotland. Scotland's Census 2011. table K2310SC ethnic group. Available: https://www.scotlandscensus.gov. uk/census-results [Accessed 06 Jun 2019].

11 Census Office for Northern Ireland. 2011 census in Northern Ireland. ethnic group full detail: QS201NI. Available: https://www.nisra.gov. uk/statistics/2011-census/results[Accessed 06 Jun 2019].

12 Ranganathan M, Bhopal R. Exclusion and inclusion of nonwhite ethnic minority groups in 72 North American and European cardiovascular cohort studies. PLoS Med 2006;3:e44.

13 Wells GA, Shea B, O'Connell D, et al. The Newcastle-Ottawa scale (NOS) for assessing the quality if nonrandomized studies in meta-analyses. Available: http://www.ohri.ca/programs/clinical_ epidemiology/oxford.htm [Accessed 05 Aug 2019].

14 Razum O, Zeeb H, Rohrmann S. The 'healthy migrant effect'--not merely a fallacy of inaccurate denominator figures. Int $\mathrm{J}$ Epidemiol 2000;29:191-2.

15 Bhopal RS. Migration, ethnicity, race, and health in multicultural societies. 2 edn. Oxford: Oxford University Press, 2014.

16 Guyatt GH, Oxman AD, Vist GE, et al. Grade: an emerging consensus on rating quality of evidence and strength of recommendations. $B M J$ 2008;336:924-6.

17 Borenstein M, Hedges LV, Higgins JPT, et al. A basic introduction to fixed-effect and random-effects models for meta-analysis. Res Synth Methods 2010;1:97-111.

18 Perneger TV. Estimating the relative hazard by the ratio of logarithms of event-free proportions. Contemp Clin Trials 2008;29:762-6.

19 Jones ME, Swerdlow AJ. Bias in the standardized mortality ratio when using general population rates to estimate expected number of deaths. Am J Epidemiol 1998;148:1012-7.

20 Tsai SP, Wen CP. A review of methodological issues of the standardized mortality ratio (SMR) in occupational cohort studies. Int J Epidemiol 1986;15:8-21.

21 Egger M, Davey Smith G, Schneider M, et al. Bias in meta-analysis detected by a simple, graphical test. BMJ 1997;315:629-34.

22 Lau J, Antman EM, Jimenez-Silva J, et al. Cumulative metaanalysis of therapeutic trials for myocardial infarction. $N$ Engl $J$ Med 1992;327:248-54.

23 Higgins JPT, Altman DG, Sterne JAC. Chapter 9: Analysing data and undertaking meta-analysis. In: Higgins JP, Green S, eds. Cochrane Handbook for systematic reviews of interventions version 5.1.0. The Cochrane Collaboration, 2011. 